\title{
Differences in renal stone treatment and outcomes for patients treated either with or without the support of a ureteral access sheath: The Clinical Research Office of the Endourological Society Ureteroscopy Global Study
}

\author{
Olivier Traxer ${ }^{1} \cdot$ Gunnar Wendt-Nordahl $^{2} \cdot$ Hiren Sodha $^{3} \cdot$ Jens Rassweiler $^{4}$.

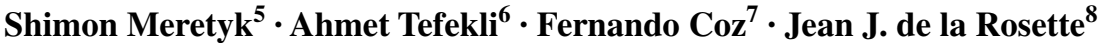

Received: 15 January 2015 / Accepted: 28 April 2015 / Published online: 14 May 2015

(c) The Author(s) 2015. This article is published with open access at Springerlink.com

\begin{abstract}
Purpose To describe the differences in the treatment and the outcomes of renal stones treated with flexible ureteroscopy (URS) either with or without the support of a ureteral access sheath (UAS).

Methods The Clinical Research Office of the Endourological Society URS Global Study involved the collection of prospective data from consecutive patients treated with URS at centers around the world over a 1-year period. Baseline characteristics, stone location, treatment details, postoperative outcomes and complications were recorded. Inverse-probability-weighted regression adjustment (IPWRA) analyses were conducted on outcome from
\end{abstract}

On behalf of the CROES URS Global Study Group.

Jean J. de la Rosette

j.j.delarosette@amc.uva.nl

1 Department of Urology, University Pierre et Marie Curie, Paris, France

2 Department of Urology, Sindelfingen-Böblingen Medical Center, Sindelfingen, Germany

3 Department of Urology, RG Stone Urology and Laparoscopy Hospital, Andheri, Mumbai, India

4 Department of Urology, SLK-Klinikum am Gesundbrunnen, Heilbronn, Germany

5 Department of Urology, Rambam Medical Center, Faculty of Medicine, Technion-Israel Institute of Technology, Haifa, Israel

6 Department of Urology, School of Medicine, Bahcesehir University, Istanbul, Turkey

7 Department of Urology, Hospital Militar, Universidad de Los Andes, Santiago, Chile

8 Department of Urology, AMC University Hospital, Meibergdreef 9, 1105 AZ Amsterdam, The Netherlands patients treated with or without the use of a UAS to determine the impact on stone-free rates (SFRs).

Results Of 2239 patients treated with flexible URS, 1494 (67\%) patients were treated with the use of a UAS and 745 (33\%) without a UAS. The IPWRA analyses conducted on 1827 patients with complete data and based on treatment and outcome models showed that if URS procedures were performed without the use of an UAS, the average stonefree rate would be 0.504 compared with 0.753 with a UAS. This average treatment effect of 0.248 was not significant $(P=0.604)$. Using IPWRA analysis on only the treated population in the estimations revealed no significant difference between using and not using a UAS (31\%; ATET: $0.311 ; P=0.523)$.

Conclusions The study showed no difference in SFR when a UAS was used or not. Whereas UAS did not increase the risk of ureteral damage or bleeding, postoperative infectious complications were reduced.

Keywords Ureteroscopy $\cdot$ Renal stones $\cdot$ Ureteral access sheath treatment outcome $\cdot$ Complications

\section{Introduction}

The ureteral access sheath (UAS) has been developed on the same concept as AMPLATZ sheath for percutaneous nephrolithotomy, i.e., to allow direct access to the kidney and to decrease the intra-renal pressure during upper-tract endourological procedures. It has become increasingly popular as it offers a number of potential advantages including facilitation of access to the renal collecting systems, multiple entry and reentry, decreased intra-renal pressure, and improved drainage around the scope [1-5]. The UAS has the added advantage of allowing passive elimination of 
small stone fragments generated during laser fragmentation with the exit of irrigation fluid. Nevertheless, the use of the UAS can itself cause damage to the ureter by over distension which compromises ureteral blood flow, resulting in ureteral ischemia [6] or direct damage to the ureter during the insertion of the UAS [7].

The CROES initiated the Ureteroscopy (URS) Global Study to establish a prospective global database to examine the worldwide use of URS and determine factors affecting outcome. In the currently presented study, inverse-probability-weighted regression adjustment (IPWRA) is used to describe differences in stone-free rate (SFR) of patients who underwent URS treatment for their renal stones, with or without the use of a UAS.

\section{Patients and methods}

\section{Study population}

The URS Global Study is a prospective, observational, international, multicenter study with data collected on consecutive patients treated with URS over a 1-year period at each participating center. The overall study period was from January 2010 until October 2012. Centers were asked to treat patients according to their local protocols. Institutional Research Board (IRB) or Institutional Ethics Committee approval was obtained by all participating centers before the start of the study. If IRB was not needed, the centers followed the protocol according the rules of Good Clinical Practice.

Patients who were eligible for inclusion in the present analysis were those who were candidates for flexible URS for renal stones as a primary treatment or after failure of a previous treatment and aged $\geq 18$ years. No specific exclusion criteria were applied. Details of treatment including secondary treatment and patient follow-up have been described previously [8].

\section{Assessment}

Data were encrypted and collected electronically through a web-based Web site: www.croesoffice.org and held in a central database at the CROES office. Data included patient epidemiological characteristics, calculus specification, type of treatment, and postoperative outcomes and complications. Stone burden was calculated as the sum of all stone sizes (length $\times$ width $\times 0.25 \times 3.14159$ ). The classification of a patient being stone free (SFR) was based on the absence of stones or fragments $>1 \mathrm{~mm}$. Operating time was defined as the time from the insertion of the endoscope until the insertion of a bladder catheter. Treatment failure was defined as stone still in situ, remaining stone fragments
$>1 \mathrm{~mm}$, and failed access. Treatment success was defined as a patient free of stones $>1 \mathrm{~mm}$.

\section{Confounders}

Preoperative possible confounders were body mass index (BMI), age, gender, stone location, stone size, stone burden, American Society of Anesthesiologists (ASA) score, preoperative stent placement, anticoagulant and antibiotics use, comorbidities such as diabetes, cardiovascular disease (CVD), prednisone use, Crohn's disease, renal congenital abnormalities, a solitary kidney, case volume and academic status of the operating center. Intra-operative possible confounders were type or URS, type of fragmentation device, operation time, intra-operative complications and a postprocedural stent placement. Postoperative possible confounders were the method of evaluation, the length of hospital stay, retreatment, readmission and postoperative complications.

\section{Statistical analysis}

Inverse-probability-weighted regression adjustment (IPWRA) analyses were performed to determine whether there was a difference in SFR between using and not using of a UAS, independent from well-known possible confounders. Inversed probability weighting (IPW) is a method in which each observation is weighted by the propensity score of individual observations. If a subject had a higher probability of being in a group (using UAS or not), it was considered as overrepresented and therefore was given a lower weight. Alternatively, if the patient had a smaller probability of being in the group, it was considered as underrepresented and was given a higher weight. Generally, in an observational study, the weighting adjustment removes sampling bias [9]. Subsequently, IPWRA is a regression analysis in which preoperative characteristicsbased inverse probability weights are used in a regression model describing the relationship of interest.

\section{Results}

\section{Patient characteristics}

A total of 2239 patients with renal stones were treated with flexible URS. Of these $1494(67 \%)$ patients were treated with the use of a UAS and $745(33 \%)$ without a UAS. Baseline characteristics are shown in Table 1. Overall, subjects treated with an UAS have higher preoperative ASA scores and larger stones.

Additionally, the UAS was most often used in North America (71.9\%), and South America (76.1\%), compared 
Table 1 Preoperative characteristics of patients with renal stones treated with a flexible ureteroscopy (URS) with or without the assistance of a ureteral access sheath (UAS)

\begin{tabular}{|c|c|c|c|c|}
\hline Parameter & $\begin{array}{l}\text { Patients treated with UAS } \\
(n=1494)\end{array}$ & $\begin{array}{l}\text { Patients treated without UAS } \\
(n=745)\end{array}$ & $\begin{array}{l}\text { Total population } \\
(n=2239)\end{array}$ & Group difference statistic \\
\hline Age (years), mean (SD) & $\begin{array}{l}51.2(14.98) \\
(n=1494)\end{array}$ & $\begin{array}{l}50.2(14.95) \\
(n=745)\end{array}$ & $\begin{array}{l}50.9(14.98) \\
(n=2239)\end{array}$ & NS \\
\hline \multicolumn{5}{|l|}{ Gender } \\
\hline Male, $n(\%)$ & $901(60.3)$ & $462(62.0)$ & $1363(60.9)$ & \\
\hline Female, $n(\%)$ & $\begin{array}{l}593(39.7) \\
(n=1494)\end{array}$ & $\begin{array}{l}283(38.0) \\
(n=745)\end{array}$ & $\begin{array}{l}876(39.1) \\
(n=2239)\end{array}$ & NS \\
\hline BMI, mean (SD) & $\begin{array}{l}27.8(7.4) \\
(n=1398)\end{array}$ & $\begin{array}{l}27.4(5.8) \\
(n=606)\end{array}$ & $\begin{array}{l}27.7(6.93) \\
(n=2004)\end{array}$ & NS \\
\hline \multicolumn{5}{|l|}{ Comorbidity, $n(\%)$} \\
\hline $\mathrm{DM}$ & $\begin{array}{l}192(12.9) \\
(n=1483)\end{array}$ & $\begin{array}{l}100(13.7) \\
(n=729)\end{array}$ & $\begin{array}{l}292(13.2) \\
(n=2212)\end{array}$ & NS \\
\hline CVD & $\begin{array}{l}503(34.0) \\
(n=1479)\end{array}$ & $\begin{array}{l}193(26.5) \\
(n=729)\end{array}$ & $\begin{array}{l}696(31.5) \\
(n=2208)\end{array}$ & NS \\
\hline Prednisone & $\begin{array}{l}28(1.9) \\
(n=1479)\end{array}$ & $\begin{array}{l}6(0.8) \\
(n=730)\end{array}$ & $\begin{array}{l}34(1.5) \\
(n=2208)\end{array}$ & NS \\
\hline Crohn's disease & $\begin{array}{l}15(1.0) \\
(n=1479)\end{array}$ & $\begin{array}{l}13(1.8) \\
(n=731)\end{array}$ & $\begin{array}{l}28(1.3) \\
(n=2210)\end{array}$ & NS \\
\hline Anticoagulation & $\begin{array}{l}153(10.4) \\
(n=1475)\end{array}$ & $\begin{array}{l}57(7.8) \\
(n=729)\end{array}$ & $\begin{array}{l}210(9.5) \\
(n=2204)\end{array}$ & NS \\
\hline Antibiotics & $\begin{array}{l}1312(88.8) \\
(n=1478)\end{array}$ & $\begin{array}{l}662(91.7) \\
(n=722)\end{array}$ & $\begin{array}{l}1974(89.7) \\
(N=2200)\end{array}$ & NS \\
\hline \multicolumn{5}{|l|}{ Renal stone location, $n(\%)$} \\
\hline Upper pole & $115(7.9)$ & $63(8.6)$ & $178(8.1)$ & NS \\
\hline Mid pole & $118(8.1)$ & $78(10.6)$ & $196(9.0)$ & \\
\hline Lower pole & $505(34.9)$ & $271(36.8)$ & $776(35.5)$ & \\
\hline Renal pelvis & $253(17.5)$ & $130(17.7)$ & $383(17.5)$ & \\
\hline Multiple & $\begin{array}{l}458(31.6) \\
(n=1449)\end{array}$ & $\begin{array}{l}194(26.4) \\
(n=722)\end{array}$ & $\begin{array}{l}652(29.8) \\
(n=2082)\end{array}$ & \\
\hline \multicolumn{5}{|l|}{ Stone size, $n(\%)$} \\
\hline$<10 \mathrm{~mm}$ & $720(51.9)$ & $458(65.8)$ & $1178(56.6)$ & $P<0.01$ \\
\hline$\geq 10 \mathrm{~mm}$ & $\begin{array}{l}666(48.1) \\
(n=1386)\end{array}$ & $\begin{array}{l}238(34.2) \\
(n=696)\end{array}$ & $\begin{array}{l}904(43.4) \\
(n=2082)\end{array}$ & \\
\hline Stone burden, mean $(\mathrm{SD})^{\mathrm{a}}$ & $\begin{array}{l}108.3(114.4) \\
(n=1287)\end{array}$ & $\begin{array}{l}99.2(100.5) \\
(n=658)\end{array}$ & $\begin{array}{l}102.2(109.6) \\
(n=1945)\end{array}$ & NS \\
\hline Previous calculus treatment, $n(\%)$ & $\begin{array}{l}765(51.8) \\
(n=1476)\end{array}$ & $\begin{array}{l}316(42.8) \\
(n=739)\end{array}$ & $\begin{array}{l}1081(48.8) \\
(n=2215)\end{array}$ & NS \\
\hline PCNL & $\begin{array}{l}206(14.0) \\
(n=1476)\end{array}$ & $\begin{array}{l}58(7.8) \\
(n=738)\end{array}$ & $\begin{array}{l}264(11.9) \\
(n=2218)\end{array}$ & \\
\hline SWL & $\begin{array}{l}408(27.7) \\
(n=1473)\end{array}$ & $\begin{array}{l}163(22.1) \\
(n=738)\end{array}$ & $\begin{array}{l}571(25.8) \\
(n=2211)\end{array}$ & \\
\hline Ureterolithotomy & $\begin{array}{l}11(0.7) \\
(n=1479)\end{array}$ & $\begin{array}{l}10(1.3) \\
(n=741)\end{array}$ & $\begin{array}{l}21(0.9) \\
(n=2220)\end{array}$ & \\
\hline URS & $\begin{array}{l}384(26.0) \\
(n=1475)\end{array}$ & $\begin{array}{l}168(22.6) \\
(n=742)\end{array}$ & $\begin{array}{l}552(24.9) \\
(n=2217)\end{array}$ & \\
\hline Pyelolithomy & $\begin{array}{l}25(1.7) \\
(n=1484)\end{array}$ & $\begin{array}{l}10(1.3) \\
(n=742)\end{array}$ & $\begin{array}{l}35(1.6) \\
(n=2226)\end{array}$ & \\
\hline UPJ pyeloplasty & $\begin{array}{l}12(0.8) \\
(n=1479)\end{array}$ & $\begin{array}{l}4(0.5) \\
(n=740)\end{array}$ & $\begin{array}{l}16(0.7) \\
(N=2222)\end{array}$ & \\
\hline Solitary kidney & $\begin{array}{l}46(3.1) \\
(n=1482)\end{array}$ & $\begin{array}{l}19(2.6) \\
(n=740)\end{array}$ & $\begin{array}{l}65(2.9) \\
(n=2222)\end{array}$ & NS \\
\hline
\end{tabular}


Table 1 continued

\begin{tabular}{|c|c|c|c|c|}
\hline Parameter & $\begin{array}{l}\text { Patients treated with UAS } \\
(n=1494)\end{array}$ & $\begin{array}{l}\text { Patients treated without UAS } \\
(n=745)\end{array}$ & $\begin{array}{l}\text { Total population } \\
(n=2239)\end{array}$ & Group difference statistic \\
\hline Renal congenital abnormality, $n(\%)$ & $105(7.0)$ & $40(6.4)$ & $145(6.5)$ & NS \\
\hline Horse shoe & $18(1.2)$ & $4(0.5)$ & $22(1.0)$ & \\
\hline Ectopic & $4(0.3)$ & $4(0.5)$ & $8(0.4)$ & \\
\hline Malrotation & $\begin{array}{l}4(0.3) \\
(n=1490)\end{array}$ & $\begin{array}{l}3(0.4) \\
(n=741)\end{array}$ & $\begin{array}{l}7(0.3) \\
(n=2231)\end{array}$ & \\
\hline Preoperative stent, $n(\%)$ & $533(35.8)$ & $284(38.4)$ & $816(36.7)$ & NS \\
\hline Double J & $511(95.9)$ & $278(98.2)$ & 789 (96.7) & \\
\hline Single J & $8(1.5)$ & - & $8(1.0)$ & \\
\hline Ureteric catheter & $\begin{array}{l}3(0.6) \\
(n=1487)\end{array}$ & $\begin{array}{l}2(0.7) \\
(n=739)\end{array}$ & $\begin{array}{l}5(0.6) \\
(n=1432)\end{array}$ & \\
\hline \multicolumn{5}{|l|}{ ASA score, $n(\%)$} \\
\hline $\mathrm{I}$ & $512(36.4)$ & $267(41.4)$ & $779(38.0)$ & $P \leq 0.01$ \\
\hline II & $649(46.2)$ & $266(41.2)$ & $915(44.6)$ & \\
\hline III & $228(16.2)$ & $105(16.3)$ & $333(16.2)$ & \\
\hline IV & $\begin{array}{l}16(1.1) \\
(n=1405)\end{array}$ & $\begin{array}{l}7(1.1) \\
(n=645)\end{array}$ & $\begin{array}{l}23(1.1) \\
(n=2050)\end{array}$ & \\
\hline \multicolumn{5}{|l|}{ Case volume, $n(\%)$} \\
\hline Low-volume center & $314(21.0)$ & $149(20.0)$ & $463(20.7)$ & NS \\
\hline High-volume center & $\begin{array}{l}1180(79.0) \\
(n=1487)\end{array}$ & $\begin{array}{l}596(80.0) \\
(n=745)\end{array}$ & $\begin{array}{l}1776(79.3) \\
(n=2082)\end{array}$ & \\
\hline
\end{tabular}

Sample sizes for which data were available are shown in parenthesis

Group differences were tested with a $t$ test for continuous variables, a Chi-square test for dichotomous and an ANOVA for categorical variables $B M I$ body mass index, $D M$ diabetes mellitus, $C V D$ cardiovascular disease, $P C N L$ percutaneous nephrolithotomy, $S W L$ extracorporeal shockwave lithotripsy, URS ureteroscopy, UPJ ureteropelvic junction, ASA American Society of Anesthesiologists

${ }^{a}$ Stone burden was calculated as the sum of all stone sizes (length $\times$ width $\times 0.25 \times 3.14159$ )

with Asia (67.3\%), Western Europe (66.1\%) and Eastern Europe $(58.3 \%)$. Systematic use $(\geq 80 \%$ of the procedures) of a UAS occurred in 39 centers (47 \%), and never in 14 centers $(17 \%)$.

\section{Operating parameters}

Intra-operative characteristics are shown in Table 2. More patients were treated flexible URS alone in the UAS group and more patients with flexible plus semirigid URS in the non-UAS group. Also, size of the stones is larger in the UAS group, complication rates differ between the groups, and operating time is longer in the UAS group. Laser fragmentation was the most common form of stone disruption used.

\section{Postoperative outcome and complications are shown in Table 3}

Stone-free rates were 73.3 versus $59.3 \%$ for the smaller stones and 81.5 versus $84.9 \%$ for larger stones with and without a UAS, respectively; SFRs were lower with the use of a UAS (73.9 vs. $82.8 \%$ ). Higher SFR is most outspoken in cases with multiple stone locations. Also, hospital stay is longer in cases treated with an UAS.

\section{Regression analysis}

Of the total study population, only those who had complete information ( $n=1827)$ were used in the regression analysis. The IPWRA analyses based on both treatment and outcome models are shown in Table 4. In this model, it was found that if none of the URS procedures were performed with the use of an UAS, the average stone-free rate would be 0.504 , i.e., as much as $50 \%$ of the population would be stone free. In addition, if all of the URS procedures were performed with the use of an UAS, the average stone-free rate would be 0.753 , i.e., as much as $75 \%$ of the population would be stone free. This difference of $25 \%$, however, representing the average treatment effect, was not significant (ATE: $0.248 ; P=0.604$ ). Using the IPWRA analysis on only the treated population in the estimations revealed no significant difference between using and not using of a UAS (31\%; ATET: $0.311 ; P=0.523$ ). 
Table 2 Intra-operative characteristics of patients with renal stones treated with a flexible ureteroscopy (URS) with or without the assistance of a ureteral access sheath (UAS)

\begin{tabular}{|c|c|c|c|c|}
\hline Parameter & $\begin{array}{l}\text { Patients treated with UAS } \\
(n=1494)\end{array}$ & $\begin{array}{l}\text { Patients treated without UAS } \\
(n=745)\end{array}$ & $\begin{array}{l}\text { Total population } \\
(n=2239)\end{array}$ & Group difference statistic \\
\hline Type of URS, $n(\%)$ & & & & $P<0.01$ \\
\hline Flexible & $973(65.1)$ & $416(55.8)$ & $1389(62.0)$ & \\
\hline Flexible + semirigid & $\begin{array}{l}521(34.9) \\
(n=1494)\end{array}$ & $\begin{array}{l}329(44.2) \\
(n=745)\end{array}$ & $\begin{array}{l}552(38.0) \\
(n=2239)\end{array}$ & \\
\hline \multicolumn{5}{|l|}{ Fragmentation device, $n(\%)$} \\
\hline Laser & $1304(87.9)$ & $609(82.2)$ & $1913(86.0)$ & NS \\
\hline Other & $13(0.8)$ & $16(2.1)$ & $29(1.3)$ & \\
\hline None & $\begin{array}{l}167(11.3) \\
(n=1484)\end{array}$ & $\begin{array}{l}116(15.7) \\
(n=741)\end{array}$ & $\begin{array}{l}283(12.7) \\
(n=2225)\end{array}$ & \\
\hline Operating time (mins, mean $[\mathrm{SD}]$ ) & $\begin{array}{l}80.0(44.2) \\
(n=1414)\end{array}$ & $\begin{array}{l}64.7(37.3) \\
(n=722)\end{array}$ & $\begin{array}{l}74.8(42.6) \\
(n=2136)\end{array}$ & $P \leq 0.01$ \\
\hline Stone size sub-categories, $n(\%)$ & & & & $P \leq 0.01$ \\
\hline$<10 \mathrm{~mm}$ & $720(51.9)$ & $458(65.8)$ & $1178(56.6)$ & \\
\hline$\geq 10 \mathrm{~mm}$ & $\begin{array}{l}666(48.1) \\
(n=1386)\end{array}$ & $\begin{array}{l}238(34.2) \\
(n=696)\end{array}$ & $\begin{array}{l}904(43.4) \\
(n=2082)\end{array}$ & \\
\hline Intra-operative complication, $n(\%)$ & & & & NS \\
\hline Uneventful & $1402(94.1)$ & $706(94.9)$ & $2108(94.4)$ & \\
\hline Failed & $12(0.8)$ & $13(1.7)$ & $25(1.1)$ & \\
\hline Bleeding & $31(2.1)$ & $9(1.2)$ & $40(1.8)$ & \\
\hline Perforation & $17(1.1)$ & $9(1.2)$ & $26(1.2)$ & \\
\hline Other & $5(1.0)$ & $10(1.3)$ & $30(1.3)$ & \\
\hline Converted & $3(0.2)$ & $1(0.1)$ & $4(1.2)$ & \\
\hline Avulsion & $\overline{-}(n=1490)$ & $\overline{(n}=744)$ & $\begin{array}{l}- \\
(n=2234)\end{array}$ & \\
\hline Postoperative stent, $n(\%)$ & $\begin{array}{l}1352(90.6) \\
(n=1493)\end{array}$ & $\begin{array}{l}611(82.0) \\
(n=745)\end{array}$ & $\begin{array}{l}1963(87.7) \\
(n=2238)\end{array}$ & NS \\
\hline
\end{tabular}

Sample sizes for which data were available are shown in parenthesis

Group differences were tested with a $t$ test for continuous variables, a Chi-square test for dichotomous and an ANOVA for categorical variables

\section{Discussion}

Flexible URS is currently recommended by the European Association of Urology Urolithiasis Guidelines for the treatment of renal stones sized up to $1.5 \mathrm{~cm}$ [10]. Of note, currently most urologists recommend 'dusting' kidney stones when using flexible URS rather than fragmentation and removal of fragments, especially when treating larger sized stones. Consequently, one may question differences in outcome of SFR depending on the application of fragmentation or dusting of the stone. Whereas in smaller sized stones, depending on the localization and composition either dusting or fragmentation is used, in larger sized stones often the use of dusting followed by fragmentation is applied. At the very end, the SFR is based on the fragments left following fragmentation. An UAS can be most instrumental to remove the multitude of fragments.

Reports have been made that the use of the UAS impacts on SFRs following flexible URS. L'Esperance et al. [1] conducted a retrospective review of 256 ureteroscopy procedures for the removal of renal calculi performed between 1997 and 2003 (173 with UAS and 83 without). The groups were similar in age, sex and stone burden. Overall SFRs were 79 and $67 \%$ in the UAS group and non-UAS group, respectively $(P=0.042)$. In contrast, reports from Berquet et al. [11] (86 \% in UAS group vs. $87 \%$ in non-UAS group; $n=280$ ) and Kourambas et al. [2] (78\% in the UAS groups vs. $85 \%$ in non-UAS group; $n=59$ ) reported no difference in SFRs with or without a UAS. In the current study with large patient population, SFR was $73.9 \%$ in the UAS group versus $82.8 \%$ in the non-UAS group. This indicates an UAS is not primarily used to increase SFR. However, there were a number of limitations to the study. One limitation is that the reason to use an UAS, such as fragments removal, facilitate access or decrease intra-renal pressure, was not recorded, and consequently, the primary reason for its use was unknown. Besides possible added efficacy of the use of an UAS, also the added safety of the 
Table 3 Postoperative characteristics of patients with renal stones treated with a flexible ureteroscopy (URS) with or without the assistance of a ureteral access sheath (UAS)

\begin{tabular}{|c|c|c|c|c|}
\hline Parameter & $\begin{array}{l}\text { Patients treated with access sheath } \\
(n=1494)\end{array}$ & $\begin{array}{l}\text { Patients treated without access sheath } \\
(n=745)\end{array}$ & $\begin{array}{l}\text { Total population } \\
(n=2239)\end{array}$ & \\
\hline Stone-free rate, $n(\%)$ & $\begin{array}{l}1051(73.9) \\
(n=1422)\end{array}$ & $\begin{array}{l}602(82.8) \\
(n=727)\end{array}$ & $\begin{array}{l}1653(78.0) \\
(n=2149)\end{array}$ & $P<0.01$ \\
\hline \multicolumn{5}{|l|}{ SFR by location sub-categories, $n(\%)$ : } \\
\hline Upper pole & $84(76.4)$ & $52(83.9)$ & $136(79.0)$ & NS \\
\hline Mid pole & $97(84.3)$ & $69(89.6)$ & $166(86.5)$ & NS \\
\hline Lower pole & $386(79.8)$ & $222(82.8)$ & $608(80.9)$ & NS \\
\hline Renal pelvis & $188(78.3)$ & $102(82.9)$ & $290(79.9)$ & NS \\
\hline Multiple & $\begin{array}{l}263(61.3) \\
(n=1018)\end{array}$ & $\begin{array}{l}148(79.0) \\
(n=593)\end{array}$ & $\begin{array}{l}411(66.7) \\
(n=1611)\end{array}$ & $P<0.01$ \\
\hline \multicolumn{5}{|l|}{ SFR by stone size sub-categories, $n(\%)$} \\
\hline$<10 \mathrm{~mm}$ & $118(73.3)$ & $269(59.3)$ & 387 (62.9) & NS \\
\hline$\geq 10 \mathrm{~mm}$ & $\begin{array}{l}712(81.5) \\
(n=830)\end{array}$ & $\begin{array}{l}444(84.9) \\
(n=713)\end{array}$ & $\begin{array}{l}1156(82.7) \\
(n=1543)\end{array}$ & $P \leq 0.01$ \\
\hline Method of evaluation, $n(\%)$ & & & & NS \\
\hline Ultrasound & $572(38.8)$ & $331(44.7)$ & $903(40.7)$ & \\
\hline $\mathrm{X}$-ray/KUB & $724(49.1)$ & $285(38.5)$ & $1009(45.5)$ & \\
\hline $\mathrm{CT}$ & $292(19.8)$ & $83(11.2)$ & $375(16.9)$ & \\
\hline IVU & $81(5.5)$ & $20(2.7)$ & $101(4.6)$ & \\
\hline Retrograde pyelogram & $20(1.4)$ & $66(8.9)$ & $86(3.9)$ & \\
\hline Intra-operative confirmation & $246(16.7)$ & $99(13.4)$ & $345(15.6)$ & \\
\hline Other & $16(1.1)$ & $4(0.5)$ & $20(0.9)$ & \\
\hline None & $\begin{array}{l}142(9.6) \\
(n=1476)\end{array}$ & $\begin{array}{l}29(3.9) \\
(n=741)\end{array}$ & $\begin{array}{l}171(7.7) \\
(n=2217)\end{array}$ & \\
\hline Hospital stay (d), mean (SD) & $\begin{array}{l}1.47(2.24) \\
(n=1481)\end{array}$ & $\begin{array}{l}1.69(2.92) \\
(n=738)\end{array}$ & $\begin{array}{l}1.55(2.48) \\
(n=2219)\end{array}$ & $P=0.025$ \\
\hline Retreatment, $n(\%)$ & $\begin{array}{l}142(9.5) \\
(n=1494)\end{array}$ & $\begin{array}{l}60(8.1) \\
(n=743)\end{array}$ & $\begin{array}{l}202(9.0) \\
(n=2237)\end{array}$ & NS \\
\hline Postoperative complication, $n(\%)$ & $\begin{array}{l}102(6.8) \\
(n=1494)\end{array}$ & $\begin{array}{l}40(5.4) \\
(n=745)\end{array}$ & $\begin{array}{l}142(6.8) \\
(n=2239)\end{array}$ & NS \\
\hline $\begin{array}{l}\text { Complication according to stone } \\
\text { location sub-categories, } n(\%) \text { : }\end{array}$ & & & & NS \\
\hline Upper pole & $8(7.1)$ & $5(12.5)$ & $13(8.6)$ & \\
\hline Mid pole & $12(10.7)$ & $4(10.0)$ & $16(10.5)$ & \\
\hline Lower pole & $32(28.6)$ & $12(30.0)$ & $44(28.9)$ & \\
\hline Renal pelvis & $22(19.6)$ & $10(25.0)$ & $32(21.1)$ & \\
\hline Multiple & $\begin{array}{l}38(33.9) \\
(n=112)\end{array}$ & $\begin{array}{l}9(22.5) \\
(n=40)\end{array}$ & $\begin{array}{l}47(30.9) \\
(n=152)\end{array}$ & \\
\hline $\begin{array}{l}\text { Complication according to stone size } \\
\text { sub-categories: }\end{array}$ & & & & NS \\
\hline$<10 \mathrm{~mm}$ & $52(48.6)$ & $24(60.0)$ & $76(51.7)$ & \\
\hline$\geq 10 \mathrm{~mm}$ & $\begin{array}{l}55(51.4) \\
(n=107)\end{array}$ & $\begin{array}{l}16(40.0) \\
(n=40)\end{array}$ & $\begin{array}{l}71(48.3) \\
(n=147)\end{array}$ & \\
\hline $\begin{array}{l}\text { Total postoperative complications, } \\
n(\%)\end{array}$ & $115(7.7)$ & $40(5.4)$ & $155(6.9)$ & $P=0.041$ \\
\hline Bleeding & $9(5.6)$ & $4(8.7)$ & $13(5.9)$ & \\
\hline Fever & $46(28.6)$ & $18(39.1)$ & $64(29.4)$ & \\
\hline UTI & $30(18.6)$ & $11(23.9)$ & $41(18.8)$ & \\
\hline Bladder cramps & $13(8.1)$ & $5(10.9)$ & $18(16.2)$ & \\
\hline Lung embolism & $1(0.6)$ & - & $1(0.5)$ & \\
\hline
\end{tabular}


Table 3 continued

\begin{tabular}{llll}
\hline Parameter & $\begin{array}{l}\text { Patients treated with access sheath } \\
(n=1494)\end{array}$ & $\begin{array}{l}\text { Patients treated without access sheath } \\
(n=745)\end{array}$ & $\begin{array}{l}\text { Total population } \\
(n=2239)\end{array}$ \\
\hline CVA & - & $1(2.2)$ & $1(0.5)$ \\
Sepsis & $7(4.3)$ & $7(15.2)$ & $14(6.4)$ \\
Acute abdomen & $2(1.2)$ & - & $2(0.9)$ \\
Other & $53(32.9)$ & $11(23.9)$ & $64(29.4)$ \\
& $(n=1491)$ & $(n=743)$ & $(n=2234)$ \\
Readmission $<3$ months & $180(13.1)$ & $63(9.1)$ & $243(11.8)$ \\
$n(\%)$ & $(n=1372)$ & $(n=693)$ & NS \\
\hline
\end{tabular}

Sample sizes for which data were available are shown in parenthesis

Group differences were tested with a $t$ test for continuous variables, a Chi-square test for dichotomous and an ANOVA for categorical variables $C T$ computed tomography, KUB kidneys, ureters and bladder, IVU intravenous urography, UTI urinary tract infection, CVA cardiovascular accident

Table 4 Results of the inverse-probability-weighted regression adjustment

\begin{tabular}{|c|c|c|c|c|}
\hline Stone-free status & Coefficient & SE & $P$ value & $95 \% \mathrm{CI}$ \\
\hline \multicolumn{5}{|c|}{ Potential outcome means (POM) } \\
\hline \multicolumn{5}{|l|}{ AccSh } \\
\hline No $(0) A$ & 0.504 & 0.478 & 0.291 & $-0.432,1.441$ \\
\hline Yes $(0) B$ & 0.753 & 0.012 & 0.000 & $0.730,0.776$ \\
\hline \multicolumn{5}{|c|}{ Outcome model $(0)$ for the non-treated } \\
\hline Operating time & 0.000 & 0.000 & 0.620 & $0.000,0.000$ \\
\hline $\begin{array}{l}\text { Intra-operative } \\
\text { complications }\end{array}$ & -0.306 & 2.468 & 0.901 & $-5.143,4.530$ \\
\hline $\begin{array}{l}\text { Postoperative } \\
\text { complications }\end{array}$ & 0.845 & 1.483 & 0.569 & $-2.062,3.751$ \\
\hline Constant & -0.035 & 2.088 & 0.986 & $-4.127,4.056$ \\
\hline \multicolumn{5}{|c|}{ Outcome model (1) for the treated } \\
\hline Operating time & 0.000 & 0.000 & 0.119 & $0.000,0.000$ \\
\hline $\begin{array}{l}\text { Intra-operative } \\
\text { complications }\end{array}$ & -0.237 & 0.842 & 0.779 & $-1.887,1.414$ \\
\hline $\begin{array}{l}\text { Postoperative } \\
\text { complications }\end{array}$ & -0.957 & 0.867 & 0.270 & $-2.656,0.742$ \\
\hline Constant & 1.194 & 0.069 & 0.000 & $1.058,1.329$ \\
\hline \multicolumn{5}{|l|}{ Treatment model } \\
\hline Stone burden & 0.000 & 0.001 & 0.999 & $-0.002,0.002$ \\
\hline Stone location & 0.099 & 0.039 & 0.012 & $0.022,0.176$ \\
\hline Stone size & 0.054 & 0.028 & 0.059 & $-0.002,0.110$ \\
\hline Previous treatment & 0.201 & 0.112 & 0.074 & $-0.019,0.420$ \\
\hline Academic center & -0.252 & 0.112 & 0.024 & $-0.472,-0.033$ \\
\hline Case volume & -0.002 & 0.000 & 0.000 & $-0.002,-0.001$ \\
\hline Constant & 0.370 & 0.227 & 0.103 & $-0.075,0.815$ \\
\hline
\end{tabular}

use of an UAS without increased risk of ureteral damage is important. From Table 2 in the present work one can conclude that UAS usage did not increase the risk of ureteral damage (perforation 1.1 vs. $1.2 \%$ ) nor bleeding (2.1 vs. $1.2 \%$ ), whereas Table 3 shows the reduced incidence of postoperative infectious complications (fever, UTI, sepsis $28.6,18.6$ and $4.3 \%$ vs. $39.1,23.9$ and $15.2 \%$ for the UAS vs. the non-UAS group accordingly). Noteworthy is that several UAS are marketed involving different lengths, sizes and characteristics, which relate to ureter status and anatomy. A study on compatibility of the different UASs with flexible URS showed the 12/14 F UAS to be a "universal" UAS that accepts the currently available flexible ureteroscopes [12]. But the smaller sized ureteroscopes have fueled the development of smaller sized UAS; at present $10 / 12$ and $11 / 13$ are predominantly used [12], resulting in less complications.

Another limitation may be the evaluation of SFR. We are aware that standardization of evaluation of stone management is often lacking. This has been highlighted in a recent communication on standardization on terminology following PCNL [13]. Ideally, a CT scan can best evaluate SFR following ureteroscopy. In the present real-life work, the SFR was evaluated by CT in a limited number of cases, whereas otherwise plain abdominal X-ray, renal ultrasound or intra-operative findings were used.

Finally, a more obvious limitation is the lack of randomization. As the subject characteristics between treatment groups (UAS or non-UAS) were imbalanced, the direct comparison of treatment groups was inappropriate. To overcome this, the IPWRA method [14] was used to determine whether the use an UAS impacted on SFR and correcting this for intra- and postoperative characteristics. The results showed no significant difference in SFR between the using and not using of a UAS.

In conclusion, in the URS Global Study there was no difference in SFR when a UAS was used or not. This result was independent from baseline characteristics and corrected for possible intra- and postoperative confounders. Intra-operative complication rates were favorable for the UAS group, including less ureteral damage or bleeding. 
Acknowledgments The URS Global Study was supported by an unrestricted educational grant from Boston Scientific, who had no involvement in the design, collection, analysis, interpretation or reporting of the data.

Conflict of interest No competing financial interests exist.

Ethical standard All human studies have been approved by the appropriate ethics committee and have therefore been performed in accordance with the ethical standards laid down in the 1964 Declaration of Helsinki and its later amendments.

Open Access This article is distributed under the terms of the Creative Commons Attribution 4.0 International License (http://creativecommons.org/licenses/by/4.0/), which permits unrestricted use, distribution, and reproduction in any medium, provided you give appropriate credit to the original author(s) and the source, provide a link to the Creative Commons license, and indicate if changes were made.

\section{References}

1. L'Esperance JO, Ekeruo WO, Scales CD Jr, Marguet CG, Springhart WP, Maloney ME et al (2005) Effect of ureteral access sheath on stone-free rates in patients undergoing ureteroscopic management of renal calculi. Urology 66:252-255

2. Kourambas J, Byrne RR, Preminger GM (2001) Does a ureteral access sheath facilitate ureteroscopy? J Urol 165:789-793

3. Stern JM, Yiee J, Park S (2007) Safety and efficacy of ureteral access sheaths. J Endourol 21:119-123

4. Rehman J, Monga M, Landman J, Lee DI, Felfela T, Conradie MC et al (2003) Characterization of intrapelvic pressure during ureteropyeloscopy with ureteral access sheaths. Urology 61:713-718
5. Auge BK, Pietrow PK, Lallas CD et al (2004) Ureteral access sheath provides protection against elevated renal pressures during routine flexible ureteroscopic stone manipulation. J Endourol 18:33-36

6. Lallas CD, Auge BK, Raj GV, Santa-Cruz R, Madden JF, Preminger GM (2002) Laser Doppler flowmetric determination of ureteral blood flow after ureteral access sheath placement. J Endourol 16:583-590

7. Traxer O, Thomas A (2013) Prospective evaluation and classification of ureteral wall injuries resulting from insertion of a ureteral access sheath during retrograde intrarenal surgery. J Urol 189:580-584

8. De la Rosette J, Denstedt J, Geavlete P, Keeley F, Matsuda T, Pearle M et al (2014) The clinical research office of the endourological society ureteroscopy global study: indications, complications, and outcomes in 11,885 patients. J Endourol 28:131-139

9. Xie J, Liu C (2005) Adjusted Kaplan-Meier estimator and logrank test with inverse probability of treatment weighting for survival data. Stat Med 24:3089-4010

10. Turk C, Knoll T, Petrik A, Sarica K, Skolarikos A, Straub M et al (2014) Guidelines on urolithiasis. European Association of Urology. http://www.uroweb.org/gls/pdf/22\%20Urolithiasis_LR.pdf. Accessed 9 Jan 2015

11. Berquet G, Prunel P, Verhoest G, Mathieu R, Bensalah K (2014) The use of ureteral access sheath does not improve stone-free rate after ureteroscopy for upper urinary tract stones. World J Urol 32:229-232

12. Al-Qahtani SM, Letendre J, Thomas A, Natalin R, Saussez T, Traxer O (2014) Which ureteral access sheath is compatible with your flexible ureteroscope? J Endourol 28:286-290

13. Opondo D, Gravas S, Joyce A, Pearle M, Matsuda T, Sun Y, Assimos D, Denstedt J, de la Rosette J (2014) Standardization of patient outcomes reporting in percutaneous nephrolithotomy. J Endourol 28(7):767-774

14. Sugihara M (2010) Survival analysis using inverse probability of treatment weighted methods based on the generalized propensity score. Pharm Stat 9:21-34 